\title{
A data acquisition system for unobtrusive measurement of on-road driving behavior
}

\author{
HENRY A. GELLER, DAVID K. DAMKOT, and SAM R. TOUSSIE \\ University of Vermont, Burlington, Vermont 05401
}

\begin{abstract}
Unobtrusive measurement of on-road driving utilizing permanently installed pavement sensors and movable photographic devices requires expensive installation and/or time consuming data reduction. This paper describes a mobile system for remotely measuring driving performance on public highways and recording the data in computer compatible format. Speed and lateral movements of cars are sensed via a radar antenna and video camera, electronically processed, and stored on magnetic tape. The taped records can be automatically read and compiled by computer.
\end{abstract}

In a foreward to a book which discusses the automobile insurance industry (O'Connell, 1971), Daniel P. Moynihan noted "In 1896, when there were four motor vehicles in the United States, two were in St. Louis. They managed to collide with such impact as to injure both drivers, one seriously" (p. vii). Since then, the frequency of motor vehicle accidents has increased at an alarming rate, and the resulting annual loss from these accidents is now measured in the billions of dollars for this country alone.

Early accident causation research included studies of psychology of the driver (Weiss \& Lauer, 1930), accident proneness (Bingham, 1931), attitudes and judgments (Cleveland Railway Company, 1929), and measures of driving behavior in laboratory simulators and instrumented vehicles (e.g., DeSilva \& Forbes, 1937; Forbes, 1932). Techniques were also devised to measure the performance of cars travelling on public highways (Green, 1946) to study the relationship between highway design and traffic operations. Permanent installations of electronic sensors (Byington \& Rosenbaum, 1973) and photographic devices which require visual analyses of the filmed records (Institute of Traffic Engineers, Technical Council Committee 6U, 1975) are now being used to obtain traffic flow data. Since these systems require expensive installations of sensors and data processing equipment or extensive data reduction by trained observers, traffic engineers would benefit from a more mobile and automated data collection system. Furthermore, this equipment could be used by psychologists to obtain unobtrusive performance measures for comparison with survey data obtained from selected drivers on public roads. Results from laboratory and instrumented car research could then be related to real world driving behavior and driver variables. The influences of environmental and/or personal stressors could also be examined in critical driving situations. For example, measures of

This work was supported by the Department of Transportation, National Highway Traffic Safety Administration, Contract No. 364-3-757. driving performance could be obtained at high accident locations, railroad crossings, and so on, as well as interview data concerning age, driving experience, state of intoxication, etc.

A data acquisition system which is both mobile and records performance data in computer compatible form was designed and used to study the influences of alcohol upon driving behavior on public roads (Damkot, Perrine, Whitmore, Toussie, \& Geller, 1975). Unobtrusive electronic measures of nocturnal driving performance were obtained for motorists passing selected sites on public highways. The motorists were subsequently stopped, and a research team obtained Breath Alcohol Concentration (BAC) measures as well as interview data concerning biographical variables, drinking patterns, and driving records.

\section{SYSTEM DESCRIPTION}

The data collection equipment was designed to meas ure and record speed and lateral position and to place code pulses on a magnetic tape record to designate the beginning and end of the observation window. These code pulses are computer compatible and are used during data retrieval operations to start and stop data collection. The computer program was written to start and stop the tape transport while computation was processing. Thus, the system was designed to permit virtually automatic data retrieval.

The data collection system can be described as three subsystems. The speed measurement subsystem consists of a doppler radar antenna (Kustom Signals, Inc., Model TR-6), electronic circuitry to amplify the signal, and a four-channel FM magnetic tape instrumentation recorder (Hewlett-Packard, Model 3960). The second subsystem consists of electronic circuitry which integrates the radar signal to determine a car's distance from the radar antenna and forms code pulses which are recorded on another channel of the magnetic tape. The third subsystem includes a video camera (Telemation, Model TMC-1100), 


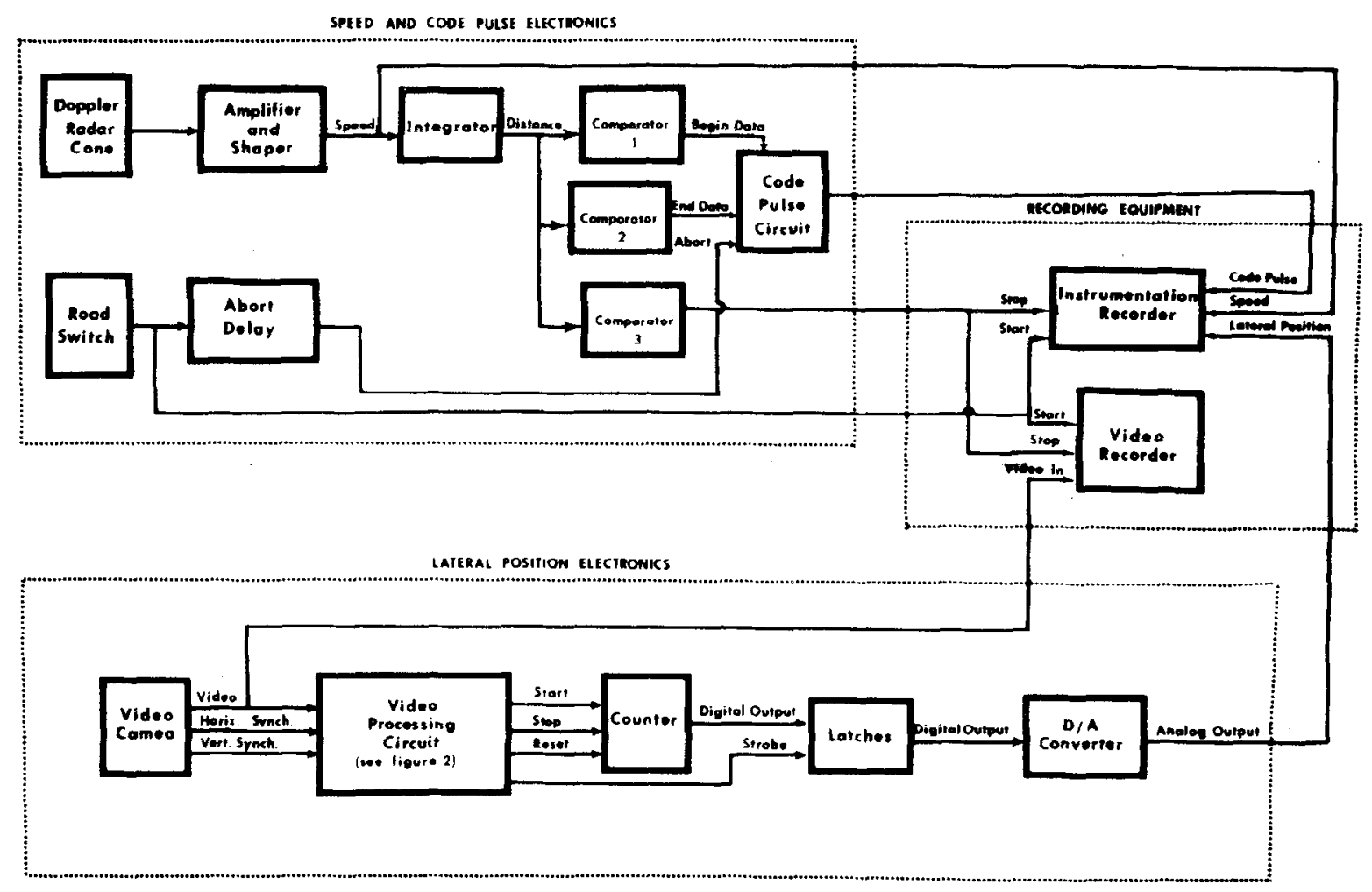

Figure 1. System flow for data acquisition, processing, and recording.

electronic circuitry for converting the video signal to an analog voltage representing lateral position, the FM magnetic tape recorder for storing this signal, and a videotape recorder (Sony, Model AV-3600) for recording the video image.

In addition to the basic electronic circuitry, subsystem function displays are used to monitor data acquisition at various points in the system. For example, an audio speaker can be connected to the radar antenna to display tone changes which occur as cars' speeds change. This is similar to the audio output available to police on their standard radar equipment. A series of lights is connected to the code pulse circuitry to permit visual monitoring of the code pulse firings. A video monitor is used to survey the image picked up by the camera and is also instrumental in aiming the camera each time it is set up. Necessary intersite comparability was accomplished by placing a light on the road edge $200 \mathrm{ft}(60.96 \mathrm{~m})$ in front of the camera and aligning the video image of the light with a sight mounted on the video monitor. This assured comparable triangulation and alignment of equipment at different road sites. We then drove a car fitted with a windshield and fender sight through the observation window and recorded the road signature. Finally, the instrumentation recorder is equipped with a meter display which can be used to check all channels to assure appropriate signal recording.

Since both the speed measurement and code pulse subsystems use the radar signal and are functionally integrated, they are described as a unit. The subsystem for measuring and recording lateral movement is described separately.

\section{Speed and Code Pulse Electronics}

This subsystem uses a stationary doppler radar antenna as a speed detector. The output of the radar antenna is a low amplitude sine wave whose frequency is proportional to the speed of the automobile being observed $(\sim 30 \mathrm{~Hz} / \mathrm{mph})$. This signal is amplified and recorded on the Hewlett-Packard instrumentation recorder to provide a continuous record of the car's speed during the time it is in the observation window. Signal amplification is necessary because the amplitude of the radar signal decreases with increasing distance of the vehicle and, at approximately $750 \mathrm{ft}(229 \mathrm{~m})$, the signal-to-noise ratio of the untreated signal is unacceptable.

The amplified signal is also integrated to provide a voltage proportional to the car's distance from the radar antenna. At specified distances, coding pulses are recorded on one channel of the recorder to specify the beginning and the end of the observation window. The integration network can be adjusted to alter the code pulse timing depending on the operating environment and length of the observation window. The maximum distance is limited by the signal-to-noise ratio and was approximately $1200 \mathrm{ft}(366 \mathrm{~m})$.

A block diagram of the system is shown in Figure 1. The speed and code pulse electronics function in the following manner. A tapeswitch is placed across the road at the location of the radar antenna. When a car trips the 
switch, the integrator begins rising as a function of speed as the car proceeds away from the radar antenna. The rate of rise for integrator output voltage is adjusted to specified feet per volt based on a calibrated electronic signal simulating $25 \mathrm{mph}$ (40.32 kph).

The output voltage of the integrator inputs a series of three comparators whose reference voltages are set to match specified distances from the radar antenna. As the integrator output reaches the reference voltage of the first comparator, the comparator output is switched, causing the code pulse board to produce a train of pulses that are recorded and signal the beginning of the data window to the computer. When the reference voltage of the second comparator is reached, the code pulse board produces a train of pulses signifying the end of the observation window. When the last comparator is switched, the integrator is stopped and reset to ground and the recorders are automatically stopped until the next car en. ters the observation window.

Since the TR- 6 antenna does not lock onto a car, the code pulse board was designed to produce a pulse train indicating aborted data if a second car entered the observation window before the first car completed the course. A short delay is provided for enabling the abort circuitry so that the rear wheels of an automobile do not cause an abort when they cross the tapeswitch. After an abort, the integrator is manually reset and the system is once again ready to record.

The code pulse board has two further provisions. An abort signal can be triggered manually to cancel a particular data run when another car enters the observation window from the opposite direction while data are being recorded. This provision is necessary because neither the radar signal nor the video signal can be locked onto a given car; thus, data records made with two vehicles in the observation window are not interpretable.

A pulse train denoting automobile braking activity is also manually triggered by an observer, A button is pushed when the brake lights come on, and again when they go off.

\section{Lateral Position Electronics}

The design of the lateral position measuring subsystem uses a fixed video camera on the road edge to view the rear lights of a car as it travels away from the camera. The rear lights appear as positive going pulses superimposed on a low voltage level background produced by the ambient nighttime light.

Damkot et al. (1975) used a video camera equipped with a 1 -in. Grade B, silicon diode array pick-up tube. The silicon diode array has several qualities which make it more desirable than a standard vidicon tube. It is capable of operating under low light conditions (faceplate illumination of $.01 \mathrm{fc}$ ), is especially sensitive to light in the near infrared range, and minimizes "bloom" and "ghosting" caused by bright moving lights.

The camera develops a complete picture by scanning horizontally from left to right. one line at a time and from top to bottom. A total of 525 lines comprise the complete picture. Every other line is scanned on each vertical sweep and on the next sweep the interstitial lines are scanned so that a particular horizontal line is written on every second vertical sweep.

The camera produces a vertical synchronization pulse at the beginning of each vertical run, and a horizontal synchronization pulse at the beginning of each horizontal scan. The frequency of the vertical synchronization pulses is $60 \mathrm{~Hz}$. Since 262.5 horizontal scans are produced for each vertical scan, the horizontal synchronization pulse frequency is $15,750 \mathrm{~Hz}$. It takes approximately 63 microseconds to complete a single horizontal scan. With the camera fixed, the time between the appearance of the horizontal synchronization pulse denoting the beginning of a horizontal scan and the positive going taillight pulse will vary as the car's lateral position varies. Thus, this time interval increases as the car moves toward the right side of the road and the camera image.

Figure 2 shows the electronic circuitry for the video signal. The incoming signal is processed through an adjustable video thresholding circuit. Appropriate adjustment of the circuit assures that the automobile taillights can be discriminated from the background light in various conditions (i.e., full moon vs. new moon; clouds vs. no clouds).

Pulses with amplitudes above the threshold value fire a monostable multivibrator (one shot) which shapes the video pulses so each has the same pulse height and width. These are compatible with the transistor-transistor logic (TTL) used in the electronics system.

Since the thresholding circuit considers any pulse above a given amplitude to be a taillight, the active screen area is reduced slightly to assure that noise spikes which appear around the synchronization pulses are not mistaken for taillights. This is accomplished by triggering serial one shots with the synchronization pulses. Thus, there is a short delay between the vertical synchronization pulse and the time when Point $A$ goes positive, determined by one shot-1 (OS1). The pulse width of one shot-2(OS2) is set to return low a short time before the next vertical synchronization pulse is due. The same process applies for the horizontal synchronization pulses.

In this manner, one of the three inputs to the triple NAND gate-1 (TN1) is held down for short intervals before and after the synchronization pulses appear. During this time period, the state of the output of the thresholding circuit will have no effect on the output of the triple NAND gate.

The time interval between the horizontal synchronization pulse and the taillight pulse is measured by a clock (ERC Model 2600-S) which starts at the beginning of the horizontal sweep and stops at the appear- 


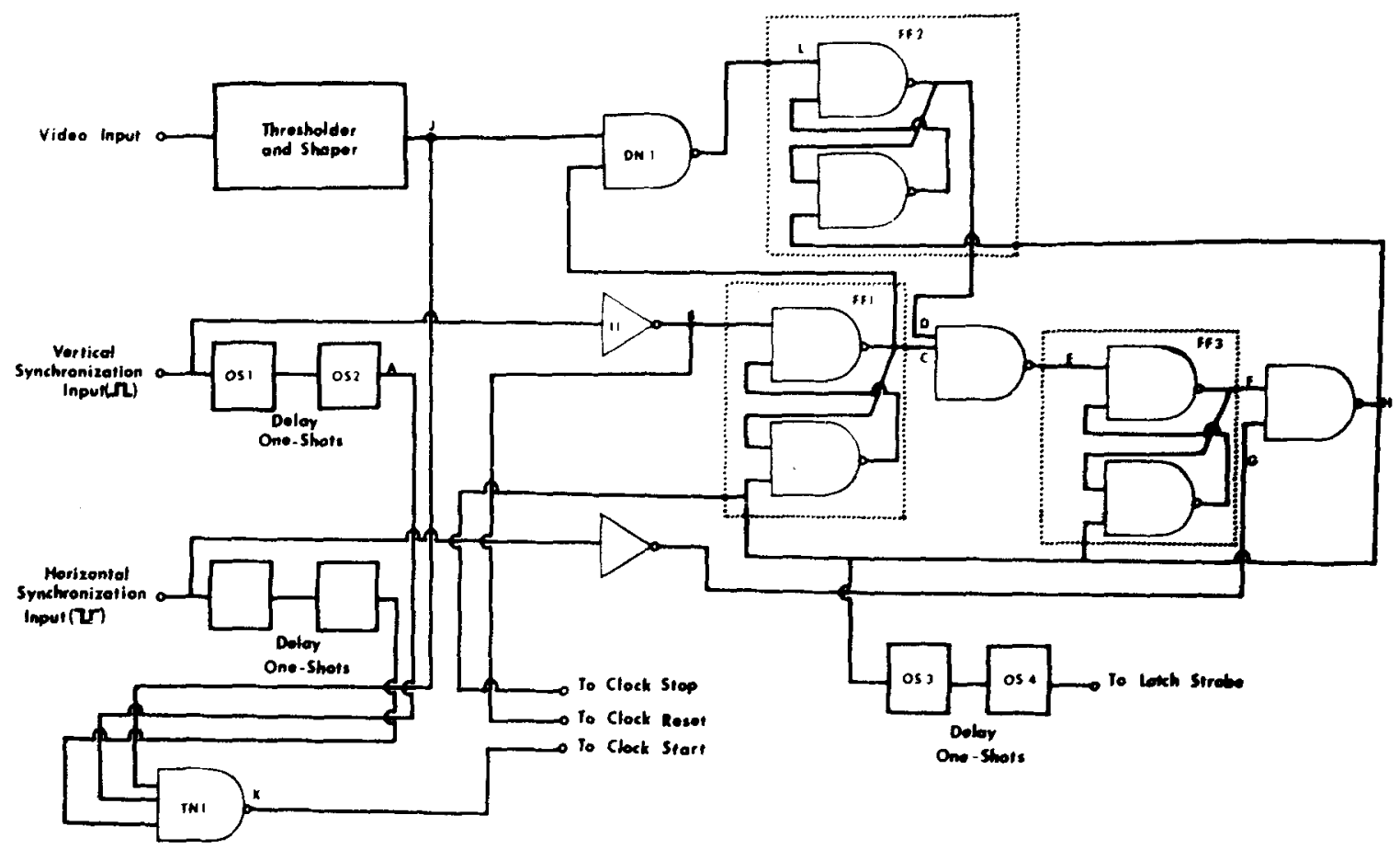

Figure 2. Video processing circuit.

ance of a taillight pulse. The clock was modified to count of $9 \mathrm{MHz}$ producing eight pulses per microsecond with the highest count ( 63 microseconds) being 504. The clock output code is Binary Coded Decimal, with 11 bits sufficing to describe the largest possible count.

The clock starts, stops, and resets on negative going TTL pulses. Once started, it stops on the first pulse to the stop input. Suceeding stop pulses have no effect, but a succeeding start pulse will restart the clock from the last count unless it is reset. When the clock has been properly reset, it will again start from zero.

The clock output is then converted to an analog signal, whose value is proportional to the time interval between the fixed viewing field edge and the taillight pulse, thereby indicating the car's lateral position. This process is accomplished once per vertical scan or 60 times per second. The analog value is updated and recorded as a function of time, and a record of the lateral position is obtained.

The typical taillight is large enough to appear on more than one horizontal video line, but the time interval need be counted only once per vertical sweep since the car will not move significantly during the $16.7 \mathrm{msec}$ needed to complete the sweep. Furthermore, recounting only adds to the total number of counts, and thus increases the amount of processing needed without adding accuracy.

The sequence of events in one vertical sweep cycle is as follows. The positive going vertical synchronization pulse goes through inverter-1 (11) causing a negative going pulse at Point $B$. This resets the clock to 0 and writes flip-flop-1 (FF1) so that Point $C$ goes positive and remains positive. At this time, Point $D$ is low, $E$ is high, $F$ and $G$ are low and $H$ is high. The horizontal synchronization pulse going negative causes Point $G$ to go positive but since $F$ is negative, $H$ will remain unchanged.

When the first positive pulse from the thresholding circuit appears (i.e., the first sweep line with taillights present), the coincidence of three positive inputs to the triple NAND gate causes Point $\mathrm{K}$ to go negative, supplying a stop pulse to the clock. These stop pulses will appear twice on every horizontal line where the taillights appear. Since Point $C$ is high, the output of dual NAND Gate-1 (DN1) at Point $L$ will go low when $J$ goes high for the first time. With both $\mathrm{C}$ and $\mathrm{D}$ high, Yoint $\mathrm{E}$ goes low, thereby writing FF3 and causing Point $F$ to go high and remain high.

On the next horizontal synchronization pulse, Point $G$ goes high, causing a negative pulse at Point $H$. This starts the clock, rewrites FF1, FF2, and FF3 and returns Points $F, D$, and $C$ to their low states to prevent the next horizontal synchronization pulse from causing start pulses at the clock. This process is inhibited until the appearance of the next vertical synchronization pulse. Finally, one shot-3 (OS3) is fired, starting a 100-microsecond delay until one-shot 4 (OS4) is fired. By this time the clock has been stopped and a count proportonal to the lateral position of the automobile is present at the clock outputs (designated counter/digital output in Figure 1).One-shot4 (OS4) strobes the latches whose 
outputs are converted into an analog signal by the digital-to-analog $(\mathrm{D} / \mathrm{A})$ converter. The result is a voltage proportional to the car's road position. This voltage will remain unchanged until the latches are strobed again during the next vertical sweep of the video camera. Thus, the car's position is updated 60 times a second and recorded on the FM tape.

\section{Video Recording}

In addition to the primary data acquisition and recording system, the video signal is simultaneously recorded on $1 / 2$ in. video tape. Both the video and instrumentation recorders are started when a car triggers the tapeswitch and are stopped when the fourth pulse code is fired. This procedure synchronizes the two machines and produces a video record that can be processed through the electronics at a later time in the event of a malfunction in the lateral position electronics.

Reprocessing the video record requires an additional synch separator since the lateral position record utilizes both the vertical and horizontal synch outputs from the camera. In other words, the video tape record can replace the video camera if a synch separator is added to the electronic circuitry and the video record is processed through the electronics as it inputs the PDP-12 computer. We have used this procedure to retrieve lateral position information for cars which were not recorded cleanly on the instrumentation tape.

\section{SYSTEM LIMITATIONS AND USES}

As originally designed, the equipment has a number of limitations that can be overcome by minor modifications of system components. These changes would produce more extensive data and also increase the mobility and utility of the system. The useful range of the equipment, as we used it, is limited by the radar antenna because the radar output becomes excessively noisy at approximately $1000 \mathrm{ft}(305 \mathrm{~m})$. This range can be extended to $2640 \mathrm{ft}(810 \mathrm{~m})$ by the addition of the signal processing unit available from the radar manufacturer (Kustom Signals, Inc., 1010 West Chestnut, Chanute, Kansas 66720: approximate cost $\$ 750$ ). This unit is the readout box used by law enforcement officers and it includes data processing circuitry which essentially ignores the signal dropouts and noise which are inherent in doppler radar signals. Extending the sensing range will require a larger lens on the video camera. We used a $100-\mathrm{mm}$ lens to obtain a resolution of \pm 6 in. $( \pm 15.24$ $\mathrm{cm})$ at $1200 \mathrm{ft}(366 \mathrm{~m})$, but a larger lens could be used to get simular resolution at $2640 \mathrm{ft}(810) \mathrm{m})$.
The mobility of the system can also be dramatically improved by changing the data collection procedures. We have found it possible to play back the video tape and, with the addition of a synch separator, obtain lateral position information from the video taped records. Since video tape recorders have an audio track, the raw video signal and the radar signal could be recorded simultaneously on the video and audio tracks of a video tape. The tapes could then be processed in the laboratory by playing them back through the electronic circuits described to input lateral position, speed, and code pulses to the computer while processing. Thus, it would be possible to collect data at roadside with a portable camera and video recorder (e.g., Sony Rover II, Model AV-3450/AVC-3400: Approximate cost \$1850) and a radar unit. Each of these pieces of equipment is available in portable models that can powered by $12 . \mathrm{V}$ $\mathrm{dc}$ sources. If these items were mounted on or in a car and they were activated by hand rather than by a tapeswitch placed on the road, it would be very easy to move from one location to another for data collection.

\section{REFERENCES}

Bingham, W. V. The prone to accident driver. Proceedings of the 17 th annual conference on highway engineering. Ann Arbor, Michigan: University of Michtgan Press. 1931.

Byington, S. R., \& Rosenbaum, M. J. The Maine Facility. Public Roads, 1973, 37, 246-255.

Cleveland Railway $\mathrm{Co}$. , and Policy Holders Service Bureau of the Metropolitan Life Insurance Co. The accident-prone employee. 1929

Damkot, D. K., Perrine, M. W., Whitmore, D. G., Toussie, S. R., \& Geller, H. A. On-the-road driving behavior and breath alcohol concentration. (Vols. I and II). National Highway Traffic Safety Administration, Technical Report DOT HS-364-3757, April, 1975.

DeSilva, H. R., \& Forbes, T. W. Driver testing results. WPA of Massachusetts, Boston, Mass. 1937.

Forbes, ' $r$. W. Measuring drivers' reactions. Personnel Journal, $1932,11,111-119$.

Green, F. H. Method for recording lateral position of vehicles. Proceedings, Highway Research Board, 1946, 26, 397-404.

Institute of Traffic Engineers. Technical Council Committee 6U,. Data collection guidlines and analy sis techniques, Part I. Traffic Engineering. 1975.45. 32-40.

$O$ 'Connell, J. The injury industry and the remedy of no-fault insurance. Chicago: Commerce Clearing House. 1971.

weiss, A. P. \& Lauer, A. R. Psychuíngical principles in automotive driving. In Ohio State Unveristy Contributions in psychology. (No. 11). Ohio State Unviersity, Columbus, Ohio, 1930 .

(Received for publication May 5, 1975; revision received September 8,1975 .) 